$02,13,19$

\title{
СИНИС-болометр с микроволновым считыванием
}

\author{
() М.А. Тарасов, С. Махашабде, А.А. Гунбина, Р.А. Юсупов, А.М. Чекушкин, С.А. Лемзяков, \\ Д.В. Нагирная, М.А. Мансфельд, В.Ф. Вдовин, В.С. Эдельман, А.С. Калабухов, Д. Винклер
}

Институт радиотехники и электроники им. В.А. Котельникова РАН, Москва, Россия

E-mail: tarasov@hitech.cplire.ru

Поступила в Редакцию 26 марта 2020 г.

В окончательной редакции 26 марта 2020 г.

Принята к публикации 2 апреля 2020 г.

Разработан, изготовлен и исследован болометр на основе структуры сверхпроводник-изоляторнормальный металл-изолятор-сверхпроводник (СИНИС), интегрированный в двойную щелевую антенну с центральной частотой $90 \mathrm{GHz}$ и соединенный со сверхпроводящим считывающим СВЧ-резонатором. Такая элементарная ячейка предназначена для многоэлементной матрицы высокочувствительного радиоастрономического приемника, в котором считывание большого числа каналов производится по одному коаксиальному кабелю вместо отдельных проводов и отдельных усилителей на каждый канал.

Ключевые слова: болометр на основе СИНИС-структуры, микроволновое считывание, частотное мультиплексирование, двойная щелевая антенна, копланарный резонатор.

DOI: 10.21883/FTT.2020.09.49763.17H

\section{1. Введение}

Активное развитие радиоастрономии субтерагерцового диапазона частот, охватывающего коротковолновую часть миллиметрового диапазона и субмиллиметрового диапазона длин волн, приводит к повышению требований, предъявляемых к приемным системам, а именно: широкий динамический диапазон и малая мощность, эквивалентная шуму (МЭШ), менее $10^{16} \mathrm{~W} / \mathrm{Hz}^{1 / 2}$ для наземных обсерваторий и на три порядка лучше для космических миссий $\left(10^{19} \mathrm{~W} / \mathrm{Hz}^{1 / 2}\right)$. В связи с этим актуальной задачей является снижение собственных шумов таких систем с целью повышения их чувствительности. Одной из весомых компонент шумовых характеристик приемной системой являются шумы, вносимые системой считывания сигналов с болометров. Применяемые ранее системы считывания на полевых транзисторах с собственными шумами на уровне $20 \mathrm{nV} / \mathrm{H}^{1 / 2}$ не имеют перспектив применения в современных приемных системах радиоастрономических обсерваторий. Создание микроволновой системы считывания для болометрических структур позволяет уйти от низкочастотного фликкер-шума, а использование криогенного усилителя в непосредственной близости от болометров снижает как шумы усилителя, так и шумы наводок на длинных проводах, чем улучшает помехоустойчивость.

C учетом этого, создание радиоастрономического приемника на основе сверхпроводникового болометра в качестве чувствительного элемента и применение частотного мультиплексирования каналов при подключении болометра к высокодобротному копланарному резонатору является актуальной задачей. Данный метод считывания позволит при необходимости считывать тысячи каналов по одной коаксиальной линии.
В качестве примера практической реализации такого подхода можно привести Simons Observatory [1] с 60000 сверхпроводящими датчиками, проект CCAT-prime [2] с 200000 датчиками, CMB Stage IV [3] с 500000 датчиками. Если предположить, что масса одного усилителя считывания составляет $1 \mathrm{~g}$, то масса на все каналы будет больше $500 \mathrm{~kg}$, при этом в криостат нужно завести миллион проводов, что делает традиционное низкочастотное считывание невозможным даже для наземных систем. Практически все современные сверхпроводниковые многоматричные приемники излучения используют мультиплексирование с частотным разделением каналов. Для количества каналов до 100 можно использовать сравнительно низкочастотные системы мегагерцового диапазона со СКВИД-усилителями [4]. Такие системы используются в инструментах POLARBEAR, EBEX, South Pole Telescope. Для большого числа каналов требуется существенно более широкий диапазон частот в несколько гигагерц. Для микроволновых детекторов на кинетической индуктивности (МКИД) созданы и успешно используются системы считывания с частотным разделением каналов. В качестве пионерских реализаций можно привести инструменты MUSIC [5], NIKA [6]. Еще одним примером такой системы является недавняя работа [7], где одновременно считывают 5000 пикселей, находящихся при температуре $250 \mathrm{mK}$. Сама система считывания подробно описана в [8].

\section{2. СИНИС-болометры с копланарными резонаторами}

В настоящей работе описан прототип интегрального приемника на основе болометров структуры сверхпро- 
Параметры четырех типов резонаторов

\begin{tabular}{c|c|c}
\hline № & $\begin{array}{c}\text { Длина копланарного } \\
\text { резонатора }\end{array}$ & $\begin{array}{c}\text { Расчетная частота } \\
\text { резонанса }\end{array}$ \\
\hline 1 & $4800 \mu \mathrm{m}$ & $6 \mathrm{GHz}$ \\
2 & $6800 \mu \mathrm{m}$ & $4.2 \mathrm{GHz}$ \\
3 & $8800 \mu \mathrm{m}$ & $3.2 \mathrm{GHz}$ \\
4 & $13800 \mu \mathrm{m}$ & $2 \mathrm{GHz}$
\end{tabular}

водник-изолятор-нормальный металл-изолятор-сверхпроводник (СИНИС) с микроволновой системой считывания сигнала на частотах до $10 \mathrm{GHz}$ и криогенным усилителем (4K) на основе транзистора с высокой подвижностью электронов (англ. НЕМТ - high electron mobility transistor) и шумовой температурой $1 \mathrm{~K}$. Достоинствами СИНИС-болометров являются широкий динамический диапазон, МЭШ не хуже $10^{16} \mathrm{~W} / \mathrm{Hz}^{1 / 2}$, высокая вольт-ваттная чувствительность (до $10^{9} \mathrm{~V} / \mathrm{W}$ ) и высокое быстродействие (менее микросекунды). Мы объединили СИНИС-болометр со сверхпроводящим резонатором считывания. Аналогичный подход был реализован в работах $[9,10]$ с СИНИС-болометром с большим объемом абсорбера, около $5 \mu \mathrm{m}^{3}$. Дизайн копланарного резонатора и двойной щелевой антенны аналогичен [11], оптимизированному для низкоомного сверхпроводникового болометра на краю резистивного перехода. В нашем случае для более высокоомного СИНИС-болометра топология копланарных линий оптимизирована численным моделированием для согласования импедансов. Первая партия образцов была изготовлена по традиционной технологии теневого напыления с висячим мостиком из резиста и длиной копланарного резонатора $4.8 \mathrm{~mm}$.

Вторая партия образцов изготовлена по безмостиковой технологии и включала четыре типа с разной длиной копланарного резонатора и расчетными частотами от 2 до $6 \mathrm{GHz}$ (таблица).

\section{3. Топология и технология изготовления}

Основу дизайна составляет одиночный болометр, интегрированный в двойную щелевую антенну с центральной частотой $90 \mathrm{GHz}$. Выбор центральной частоты $90 \mathrm{GHz}$ обусловлен тем, что первой испытательной площадкой для разработанных структур предполагается наземный Большой телескоп азимутальный (БТА) [12], а прозрачность атмосферы выше $100 \mathrm{GHz}$ в этом случае значительно хуже. Полученные результаты будут использованы для разработки прототипа приёмной системы для строящегося телескопа на плато Суффа [13] и в перспективе для отечественной космической обсерватории МИЛЛИМЕТРОН [14]. Результаты, полученные в ходе данного проекта, могут быть применимы и для других перспективных разработок, а именно: системы считывания для болометрических матриц других ти-

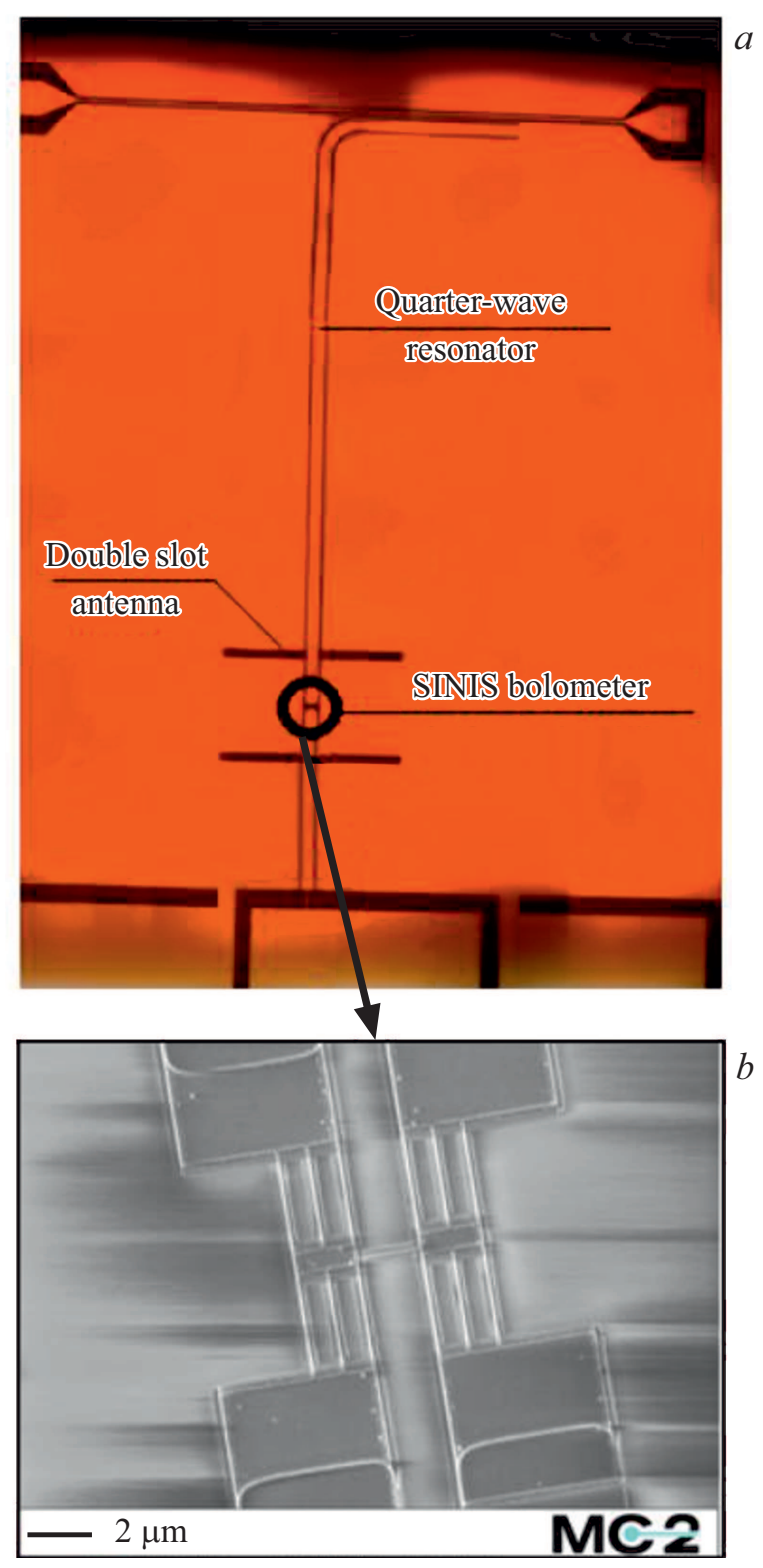

Рис. 1. a) Фотография общего вида изготовленного образца в оптическом микроскопе и $b$ ) изображение в электронном микроскопе болометрической структуры, изготовленной по технологии безмостикового теневого напыления.

пов (например, для TES и KID), СКВИД-структуры для квантово-информационных технологий, развиваемые технологии для изготовления различных микро- и наноструктур.

Планарная двойная щелевая антенна имеет хорошие характеристики направленности в сочетании с квазиоптическим рупором или диэлектрической линзой. Антенна (рис. 1) имеет две параллельных щели в нижнем слое нитрида ниобия. Эти щели когерентно возбуждаются короткими отрезками копланарных линий с СИНИС-болометром в центре. Одна секция копланарной линии проходит параллельно щели в длинной сверхпроводящей секции, которая функционирует как 


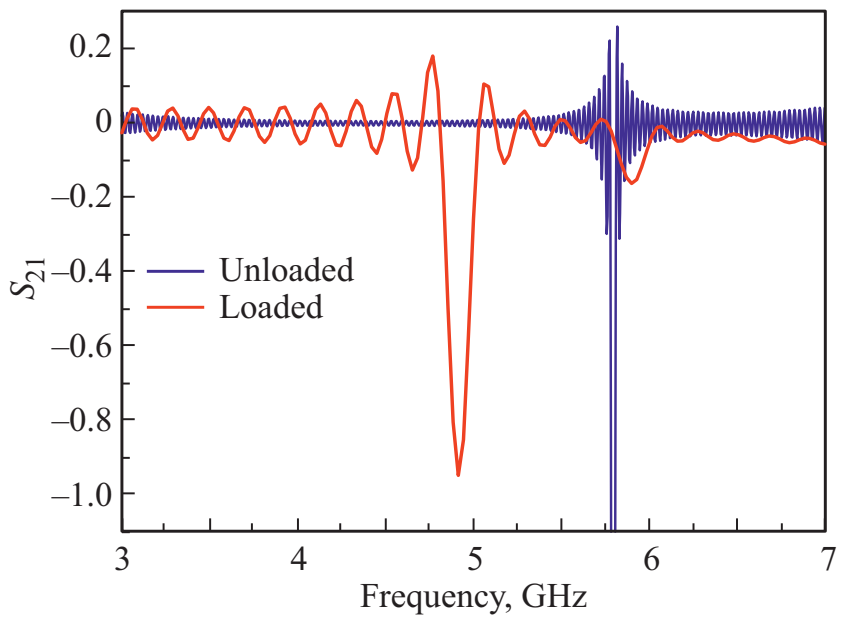

Рис. 2. Резонанс копланарного четвертьволнового резонатора без болометра, $Q=232, f=5.8 \mathrm{GHz}$, и $Q=33, f=4.95 \mathrm{GHz}$ с болометром.

четвертьволновый резонатор. Этот резонатор закорочен на дальнем конце, а разомкнутый конец замыкается СИНИС-болометром в антенне. Резонанс копланарного четвертьволнового резонатора без болометра $Q=232$, $f=5.8 \mathrm{GHz}$ и $Q=33, f=4.95 \mathrm{GHz}$ с болометром (рис. 2).

Сопротивление нормального металлического абсорбера СИНИС-болометра соответствует реальной части импеданса антенны, в которую он интегрирован. Ток смещения соответствует рабочей точке с максимальной чувствительностью.

Резонатор был изготовлен из слоя $\mathrm{NbN}$ толщиной $50 \mathrm{~nm}$ с расчетной кинетической индуктивностью $4 \mathrm{pH}$ на квадрат. Это позволяет значительно уменьшить геометрическую длину резонатора. Первый слой содержит резонатор, двойную щелевую антенну, выходной резонатор, контактные площадки и соединительные проводники. Пленка $\mathrm{NbN}$ травилась в ионной плазме $\mathrm{Cl}_{2} / \mathrm{Ar}$. Болометры были сформированы во втором слое с помощью двухслойной резистивной маски с последующим напылением под углами сверхпроводящих электродов из Al. Нормальный металл представляет собой $\mathrm{Al}$ толщиной $20 \mathrm{~nm}$, сверхпроводимость которого подавлена лежащей ниже пленкой $\mathrm{Fe}$ толщиной $1 \mathrm{~nm}$, барьер был сформирован во время окисления этого слоя $\mathrm{Fe} / \mathrm{Al}$.

Параллельно с традиционной технологией, в данной работе продолжено развитие технологии изготовления субмикронных СИН- и СИС-переходов в маске резиста без подвешенных мостиков Долана (фотография болометра, изготовленного по такой технологии - рис. $1, b$ ). Такая технология [15] имеет много преимуществ по сравнению с применяемой ранее, а именно: возможность изготовления туннельных переходов площадью от 0.01 до $1000 \mu \mathrm{m}^{2}$, улучшение точности воспроизведения размеров, улучшение электропроводности и теплопровод- ности подводящих проводников, а также возможность проводить ионную чистку подложки перед напылением. Схематичное изображение этапов новой разработанной технологии представлена на рис. 3. Суть технологии заключается в раздельном напылении двух пленок разных металлов в две ортогональные глубокие канавки в двухслойном резисте (рис. 3, $a$ и $b$ ). Напыление первой пленки вдоль первой канавки не приводит к ее напылению в ортогональную канавку (рис. 3, c), т. к. угол напыления выбран таким образом, что в направлении второй канавки напыление происходит на стенку из резиста с последующим удалением в ремувере. Аналогично и при напылении другой пленки во вторую ортогональную канавку, после поворота подложки на $90^{\circ}$ (рис. $3, d$ ). Изолятор был получен путем окисления первой пленки в напылительной камере (10 Torr, $10 \mathrm{~min})$. Применение раздельной экспозиции для двух слоев резиста позволяет точно контролировать профиль канавок и избежать образования вертикальных металлических стенок после напыления пленок.

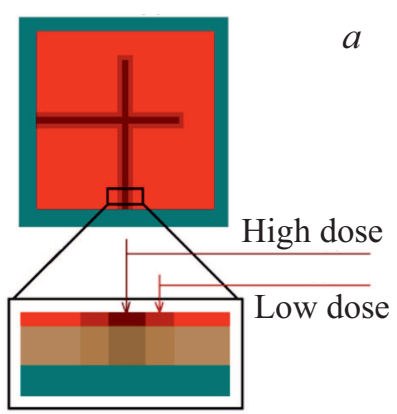

Side view

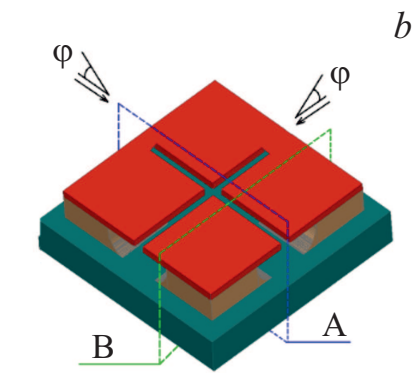

$b$
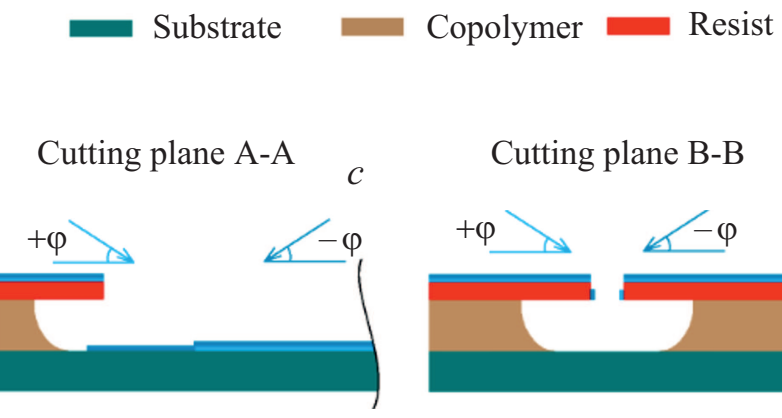

Cutting plane B-B

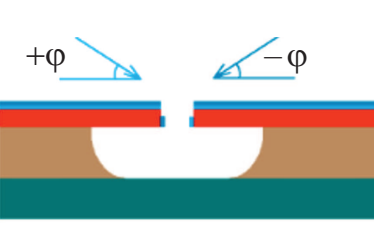

$d$
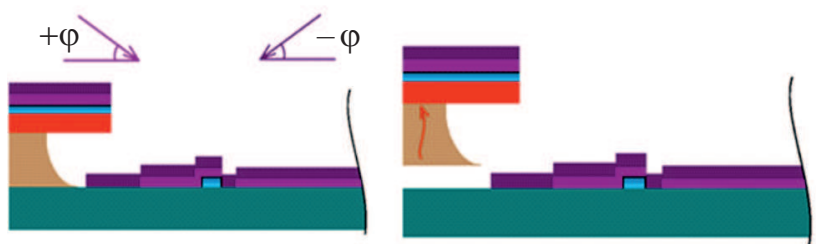

Рис. 3. Схематичное изображение этапов разработанной технологии изготовления туннельных переходов: $a$ ) экспонируемая структура до проявления; $b$ ) экспонируемая структура после проявления; $c$ ) напыление первой пленки под углами $\left.\pm 45^{\circ} ; d\right)$ напыление второй пленки в ортогональную канавку; e) удаление резиста в ремувере. 

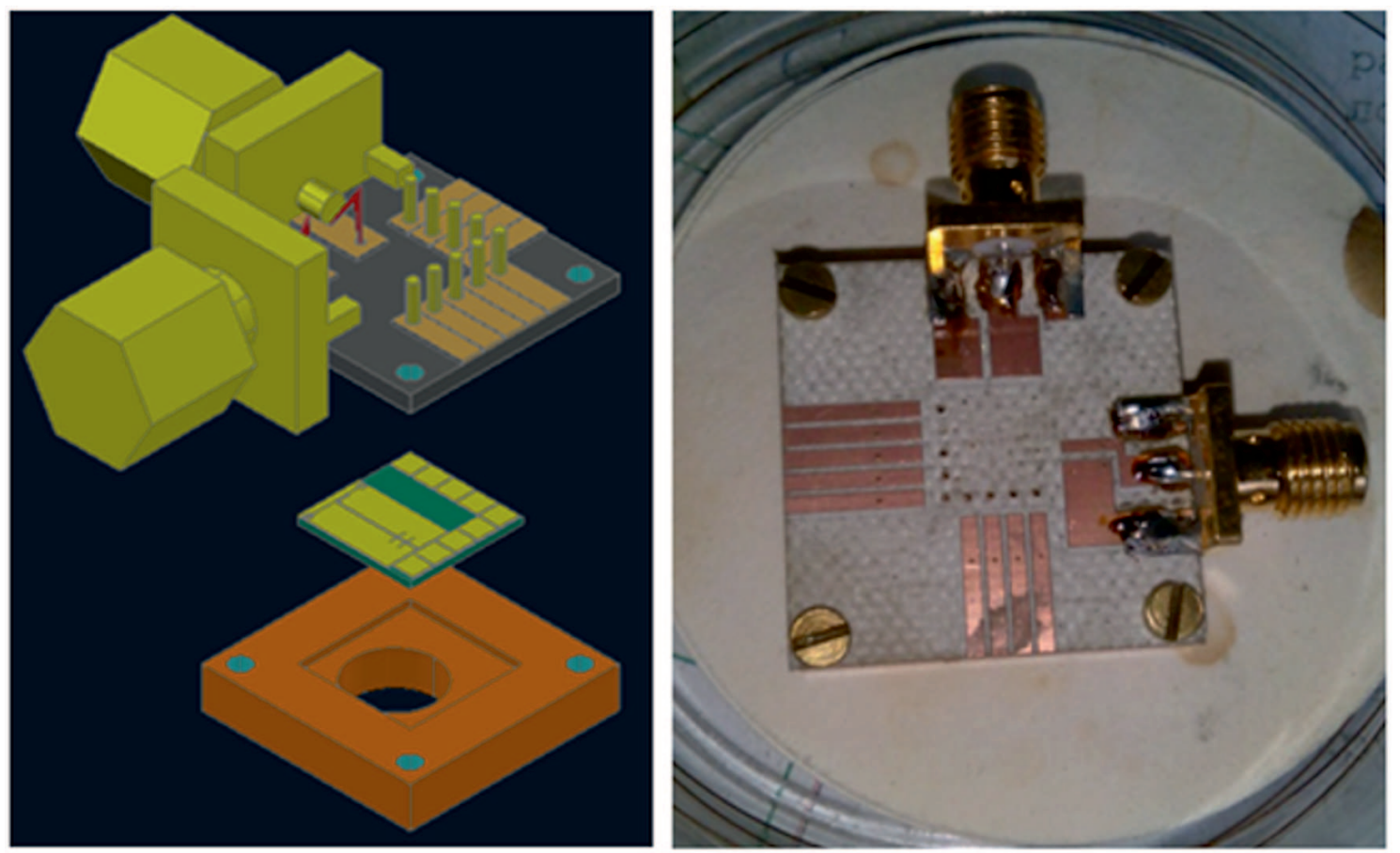

Рис. 4. Держатель образца с SMA-разъемами.

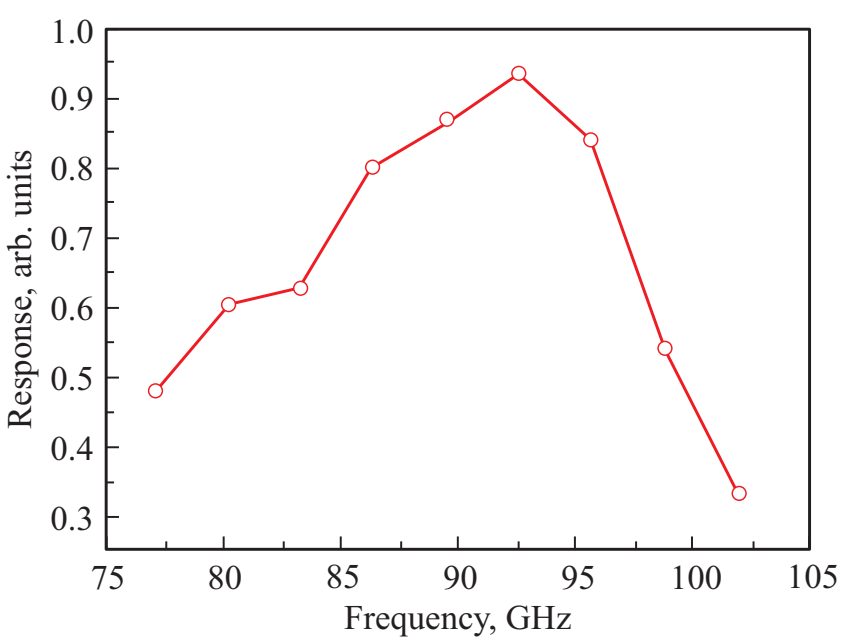

Рис. 5. Спектральный отклик болометра на излучение ЛОВ.

\section{4. Экспериментальная установка и результаты измерений}

Измерения вольт-амперных характеристик проводились в криостате фирмы Oxford Instruments Heliox AC-V при температуре $300 \mathrm{mK}$. Фотография и схематичное изображение держателя образца, разработанного для таких измерений, приведены на рис. 4. Также был измерен спектральный отклик исследуемого образца с использованием перестраиваемой лампы обратной волны (ЛОВ) в диапазоне 75-100 GHz (рис. 5). Мощность излучения нормировалась на сигнал опорного канала с пироэлектрическим приемником. Для более точной оценки спектрального отклика разработанных структур применяется дополнительная нормировка принятого сигнала на опорный сигнал (сигнал, снимаемый с рутениевого резистора, расположенного внутри криостата рядом с исследуемым образцом) и на сигнал с цепочки СИН-структур (термометра). Такая калибровка позволяет частично „исключить“ множественные переотражения внутри криостата и нагрев подложки. Особенности такой методики калибровки подробно изложены в нашей работе [16].

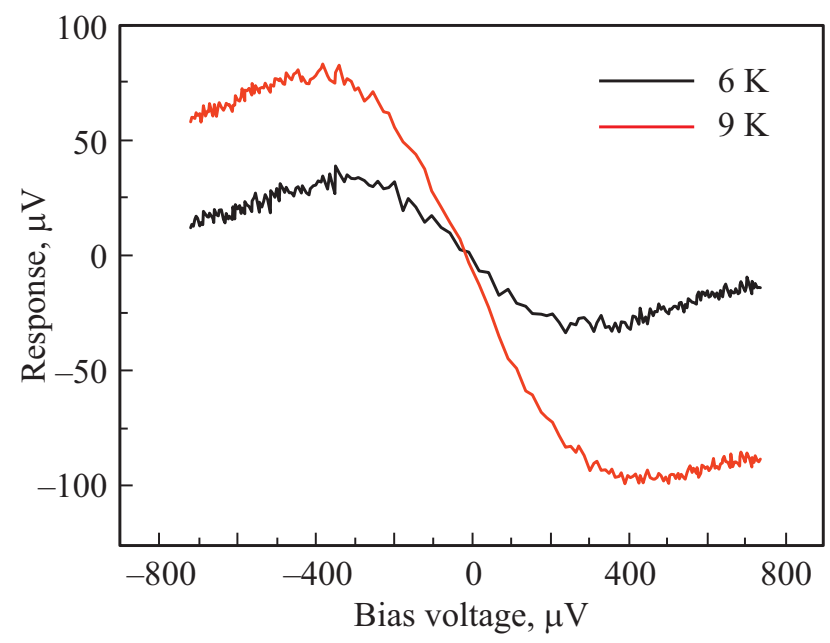

Рис. 6. Отклик на излучение черного тела при температурах 6 и $9 \mathrm{~K}$. 


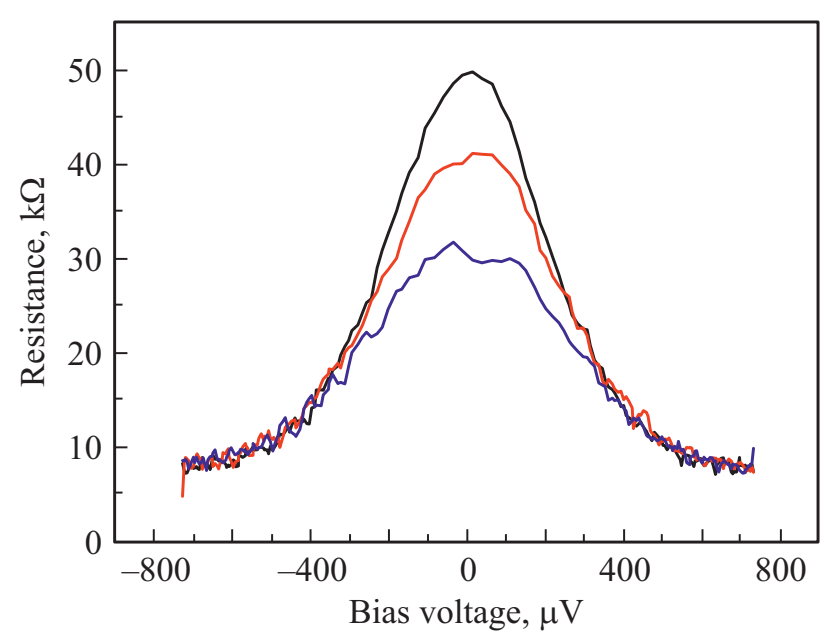

Рис. 7. Дифференциальное сопротивление при трех значениях мощности сигнала.

Мы также измерили отклик образца на излучение черного тела при температурах 6 и $9 \mathrm{~K}$ (рис. 6) в погружном криостате растворения при температуре $100 \mathrm{mK}$ [17]. Соответствующий максимум дифференциального сопротивления изменяется от 50 до $30 \mathrm{k} \Omega$. Экспериментальные значения дифференциального сопротивления при трех уровнях мощности излучения приведены на рис. 7. Для копланарной линии с сопротивлением $50 \Omega$ такая нагрузка соответствует добротности 1000 и 300 для темновых и облучаемых случаев соответственно. Изменение импеданса с 30 до $50 \mathrm{k} \Omega$ легко регистрируется по изменению резонансной частоты и добротности и позволяет проводить СВЧ-считывание с частотным разделением каналов.

\section{5. Заключение}

Был разработан, изготовлен и экспериментально исследован СИНИС-болометр, предназначенный для использования с микроволновой системой считывания. Измерен спектральный отклик на частоте сигнала в диапазоне $90 \mathrm{GHz}$. Измерен электрический отклик и дифференциальное сопротивление такого болометра на излучение черного тела при температурах 6 и $9 \mathrm{~K}$. Дифференциально-е сопротивление при этом изменяется от 50 до $30 \mathrm{k} \Omega$, что соответствует изменению добротности от 1000 до 300.

\section{Финансирование работы}

Работа выполнена в рамках государственного задания ИРЭ РАН (№ 0030-2019-0003) и государственного задания ИПФ РАН (№ 0035-2019-0005). Изготовление и исследование образцов произведено с использованием уникальной научной установки (УНУ № 352529).

\section{Конфрликт интересов}

Авторы заявляют, что у них нет конфликта интересов.

\section{Список литературы}

[1] N. Galitzki, A. Ali, K. Arnold, P. Ashton, J. Austermann, C. Baccigalupi, T. Baildon, D. Barron, J. Beall, S. Beckman. Proc. SPIE 10708, 1070804 (2018).

[2] M. Aravena, J. Austermann, K. Basu, N. Battaglia, B. Beringue, F. Bertoldi, J.R. Bond, P. Breysse, P. Bustos, S. Chapman, S. Choi, D. Chung, N. Cothard, B. Dober, C. Duell, S. Duff, R. Dunner, J. Erler, M. Fich, L. Fissel, S. Foreman, P. Gallardo, J. Gao, R. Giovanelli, U. Graf, M. Haynes, T. Herter, G. Hilton, R. Hlozek, J. Hubmayr, D. Johnstone, L. Keating, E. Komatsu, B. Magnelli, P. Mauskopf, J. McMahon, P.D. Meerburg, J. Meyers, N. Murray, M. Niemack, T. Nikola, M. Nolta, S. Parshley, R. Puddu, D. Riechers, E. Rosolowsky, S. Simon, G. Stacey, J. Stevens, J. Stutzki, A. Van Engelen, E. Vavagiakis, M. Viero, M. Vissers, S. Walker, B. Zou. arXiv:1909.02587 (2019).

[3] M. Abitbol, Z. Ahmed, D. Barron, R. Thakur, A. Bender, B. Benson, C. Bischoff, S Bryan, J. Carlstrom, C. Chang. arXiv:1706.02464 (2017).

[4] T. Lanting, H.-M. Cho, J. Clarke, M. Dobbs, A. Lee, Lueker, P. Richards, A. Smith, and H. Spieler. Nucl. Instr. Meth. Phys. Res. A 520, 548 (2004).

[5] J. Sclaerth, A. Vayonakis, P. Day, J. Glenn, J. Gao, S. Glowala, S. Kumar, H. LeDuc, B. Mazin, J. Vaillancourt, J. Zmuidzinas. J. Low Temp. Phys. 151, 684 (2008).

[6] M. Calvo, A. Benoit, A. Catalano, J. Goupy, A. Monfardini, N. Ponthieu, E. Barria, G. Bres, M. Grollier, G. Garde. J. Low Temp. Phys. 184, 816 (2016).

[7] L. Ferrari, O. Yurduseven, N. Llombart, S. Yates, J. Bueno, V. Murugesan, D. Thoen, A. Endo, A. Baryshev, J. Baselmans. IEEE TST 8, 127 (2017).

[8] J.J.A. Baselmans, J. Bueno, S.J.C. Yates, O. Yurduseven, N. Llombart, K. Karatsu, A.M. Baryshev, L. Ferrari, A. Endo, D.J. Thoen, P.J. de Visser, R.M.J. Janssen, V. Murugesan, E.F.C. Driessen, G. Coiffard, J. Martin-Pintado, P. Hargrave, M. Griffin. Astron. Astrophys. 601, A89 (2017).

[9] D.R. Schmidt, K.W. Lehnert, A.M. Clark, W.D. Duncan, K.D. Irwin, N. Miller, J.N. Ullom. Appl. Phys. Lett. 86, 053505 (2005).

[10] D.R. Schmidt, W.D. Duncan, K.D. Irwin, K.W. Lehnert, N.A. Miller, J.N. Ullom. Nucl. Instr. Meth. Phys. Res. A 559, 516 (2006).

[11] S.V. Shitov, A.A. Kuzmin, M. Merker, V.I. Chichkov, A.V. Merenkov, A.B. Ermakov, A.V. Ustinov, M. Siegel. IEEE Trans. Appl. Supercond. 27, 2100805 (2017).

[12] http://w0.sao.ru/hq/sekbta/.

[13] http://www.ipme.ru/ipme/labs/RT-70/source/start.html

[14] http://www.asc.rssi.ru/millimetron/millim.htm.

[15] М. Тарасов, А. Гунбина, Д. Нагирная, М. Фоминский. Способ изготовления устройств с тонкопленочными туннельными переходами. Заявка на патент № 2019123125 от 22.07.2019.

[16] M.A. Tarasov, A.M. Chekushkin, R.A. Yusupov, A.A. Gunbina, V.S. Edelman. J. Commun. Technol. Electron. 65, 1, 60 (2020).

[17] V.S. Edelman. Instrum. Exp. Techn. 52, 2, 301 (2009).

\section{Редактор Е.В. Толстякова}

